\title{
Hybrid-integrated version of solid-state components for terahertz frequency region
}

\author{
O.V. Zorenko ${ }^{1}$, A.V. Bychok ${ }^{1}$, T.V. Kryts'ka ${ }^{1}$, Ya.Ya. Kudryk ${ }^{2}$, V.V. Shynkarenko ${ }^{2}$ \\ ${ }^{1}$ State Enterprise Research Institute “Orion”, 8a, Eugene Pottier str., 03057 Kyiv, Ukraine \\ ${ }^{2} V$. Lashkaryov Institute of Semiconductor Physics, NAS of Ukraine \\ 41, prospect Nauky, 03680 Kyiv, Ukraine, e-mail: shynkarenko@isp.kiev.ua
}

\begin{abstract}
We present the results of development of key components for $\mathrm{THz}$ devices obtained using mathematical modeling of detector sections, radiation source and subharmonic mixers. Schottky barrier diodes with different parameters served as nonlinear elements of the above facilities.
\end{abstract}

Keywords: $\mathrm{THz}$ region, $\mathrm{SBD}$, detector, frequency multiplier, subharmonic mixer, waveguide-to-microstrip line transition, rejection filter, SPICE model.

Manuscript received 21.01.14; revised version received 24.04.14; accepted for publication 12.06.14; published online 30.06.14.

\section{Introduction}

In recent years, much consideration has been given to systems operating at $\mathrm{THz}$ frequencies. The interest to $\mathrm{THz}$ radiation was dictated by the following facts:

- this radiation is not ionizing (quanta of $0.001-0.01 \mathrm{eV})$;

- $\quad$ this radiation passes well through turbid media and fine-dispersed materials because of strong suppression of Rayleigh scattering $\left(1 / \lambda^{4}\right)$;

- the corresponding frequency region is that of molecular rotational spectrum, vibrations of biologically important DNA and proteins, and solid-state plasma vibrations;

- the photon thermal noise is almost absent in that radiation region, and sensor characteristics of detectors become better as frequency grows;

- $\quad$ at $\mathrm{THz}$ frequencies, the data receive and transfer rates as well as number of channels are much over those at radio communication.

The functional modules for $\mathrm{THz}$ region are key elements when developing novel small-sized $\mathrm{THz}$ wireless systems and technologies for radars and radio navigation, radio-wave imaging, medicine, biology, customs and anti-terrorist inspection [1-3].
In our developments, we chose microstrip lines as signal matching and isolation elements, according to the modern technologies and requirements for reduction in price of products. A Schottky barrier diode (SBD) was chosen as nonlinear element of detectors, frequency multipliers and mixers, because SBD has quadratic $I-V$ curve and the lowest loss resistance among the diodes that are applied when making microwave components [3-5].

\section{Broadband detector sections with SBD for 220-325 GHz and 325-400 GHz frequency ranges}

To exert control over the finite device of radio receiver when receiving modulated signals, the received signal should be transformed in such a way as to make the law of variation of voltage coming to input of the finite device to replicate the signal modulation law. Such a transformation of signal in radio receiver is made by a detector. Amplitude detectors are used to detect unmodulated and amplitude-modulated signals; each of them may be continuous or pulse.

The detector sections are mostly used in microwave measuring devices (in particular, in panoramic meters of standing wave ratio load), indicating devices etc. The main type of most widely used amplitude detectors is that involving a nonlinear 
element and a load to which rectified voltage is applied. Semiconductor diodes are widely used in amplitude detectors as nonlinear elements $[4,5]$.

A block diagram of detector section is presented in Fig. 1.1. A microwave signal (1) comes to an input waveguide flange (2) of detector; input signal power is supplied to a diode (4) using a waveguide-to-microstrip line tapered transition; a modulated signal envelope is removed with a rejection filter (5) to a video amplifier (6).

To develop broadband detector sections, we performed mathematical modeling for impedance characteristics of active and passive elements, microwave integrated circuit (IC) and detector sections with SBD. The models for waveguide-to-microstrip line tapered transitions of detector sections were developed using the node potential method. 3D models of waveguide-to-microstrip line transition are presented in Figs $1.2 \mathrm{a}$ and $1.2 \mathrm{~b}$.

A waveguide-to microstrip line tapered transition is the input part of detector section. The role of tapered transition is ensuring transformation of transition wave impedance over a broad frequency band and, correspondingly, bringing of microwave signal power to detector diode with minimal losses and correct configuration of electromagnetic field. The tapered transition losses are no more than $-0.5 \mathrm{~dB}$ for 220 $325 \mathrm{GHz}$ and $325-400 \mathrm{GHz}$ frequency ranges, so practically the total microwave signal power will pass the transition and come to the detector diode.

We also performed modeling and development of a low-and-high-pass filter that separates at output the frequency components lying in the given frequency band from the composite signal coming to the input. The rest of frequency components are suppressed (reflected, absorbed or branched) by the filter.

The following functions are performed by the filter in the detector section:

- it ensures virtual short circuiting at the site of detector diode connection to the filter in the operating frequency band;

- $\quad$ it serves as rejection filter RF for microwave signal;

- $\quad$ it serves as low-pass filter for a video signal;

- $\quad$ it ensures diode bias.

Shown in Figs 1.3a and 1.3b are filter topologies made on the basis of half-wave resonators with open ends. The sizes of filter elements for the $220-325 \mathrm{GHz}$ and $325-400 \mathrm{GHz}$ frequency ranges were determined using the node potential method. We also calculated frequency dependences of the transmission coefficient $\mathrm{S}_{21}$ for the topologies developed. Such filters introduce attenuation over $40 \mathrm{~dB}$ at the detector input frequency.

Based on detector diode modeling, we made detector sections for the required ranges. Shown in Figs 1.4 and 1.5 are conversion factors as function of frequency. The detector sections for the $220-325 \mathrm{GHz}$ $(325-400 \mathrm{GHz})$ frequency range ensure conversion factor within 1000-115 V/W (120-102 V/W).

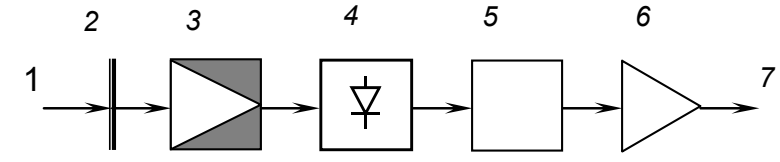

Fig. 1.1. Block diagram of detector section: 1 - microwave signal, 2 - input waveguide flange, 3 - waveguide-tomicrostrip line tapered transition, $4-\mathrm{SBD}, 5$ - rejection filter, 6 - video amplifier, 7 - output voltage.

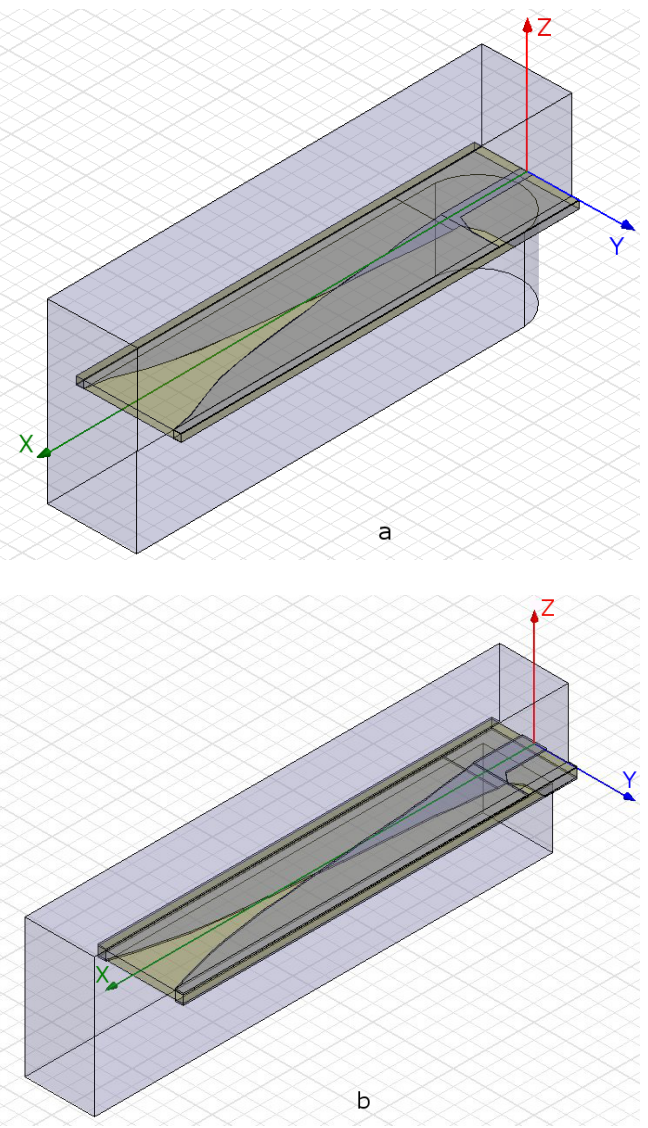

Fig. 1.2. 3D model of waveguide-to-microstrip line tapered transition: a) for a $220-325 \mathrm{GHz}$ detector section [6]; b) for a 325-400 GHz detector section.

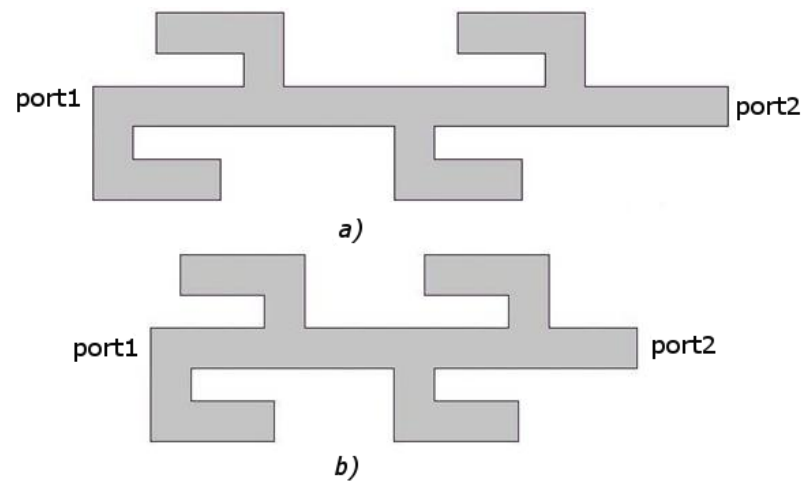

Fig. 1.3. Topology of a rejection filter: a) for $220-325 \mathrm{GHz}$ [6]; b) for 325-400 GHz. 


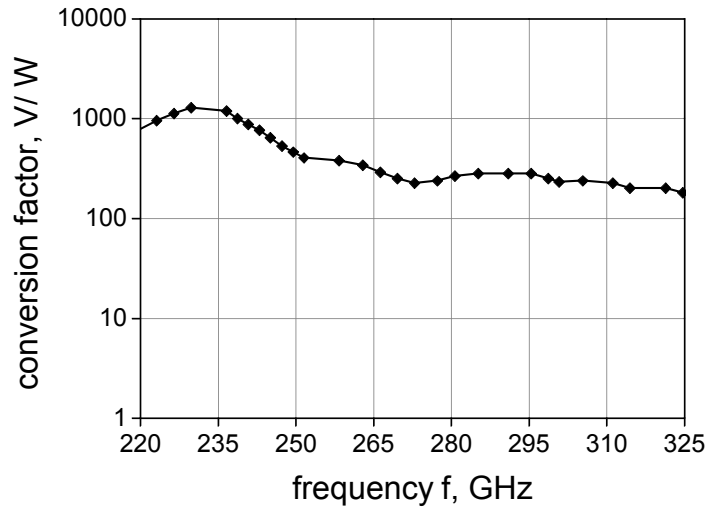

Fig. 1.4. Conversion factor of detector section as a function of frequency in the $220-325 \mathrm{GHz}$ range.

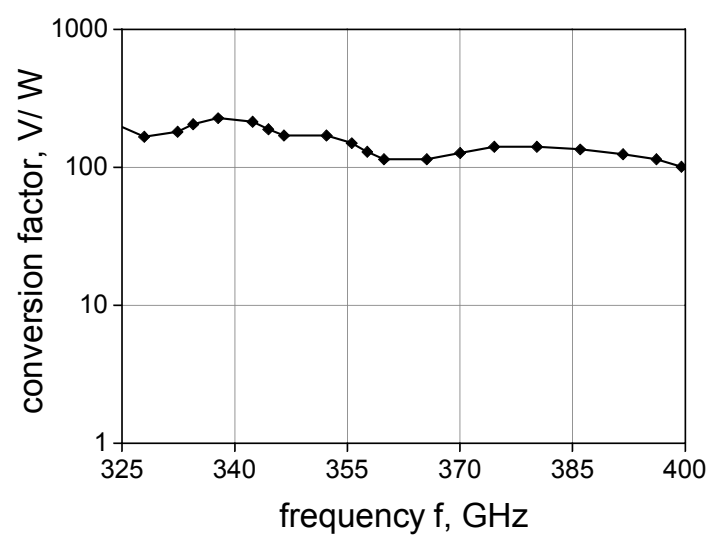

Fig. 1.5. Conversion factor of detector section as a function of frequency in the $325-400 \mathrm{GHz}$ range.

\section{Radiation sources using a frequency multiplier with SBD for $280 \mathrm{GHz}$ operating region}

To transmit a stable wanted signal at a frequency over that given by an oscillator with the required stabilization level, a frequency multiplier is used. Synthesizing of frequency spectrum also requires frequency multipliers. Their application ensures the following advantages:

- a single-frequency master-oscillator can operate at lower (by several times), thus having better stability;

- it is possible to broaden the tuning range of transmission facility, at narrower range of the single-frequency master-oscillator;

- it is possible to improve stability of device operation by decreasing feedback because input and output circuits of the frequency multiplier are tuned to different frequencies;

- a possibility to increase absolute deviation of frequency (phase) at frequency (phase) modulation appears [7].

Operation of frequency multiplier may be expressed by Fourier transform, with signal being of the form [7]:

$$
x(t)=\sum_{k=-\infty}^{\infty} c_{k} e^{i 2 \pi k f t}
$$

where the $k$-th harmonic is expressed with period $T$ :

$$
c_{k}=\frac{1}{2 \pi} \int_{0}^{T} x(t) e^{i 2 \pi k f t} d t .
$$
filter.

The required harmonic is chosen using a broadband

To develop and fabricate radiation sources based on frequency multipliers with SBD, we performed mathematical modeling of impedance and energy characteristics of active and passive elements of radiation sources. The basis for modeling is the energy diagram of metal-semiconductor contact that describes energy barrier in semiconductor near-contact layer that is responsible for potential difference in the semiconductor near-surface layer.

An active element (SBD) may be described by the Shockley equation supplemented with dependences that describe various physical effects. Modeling of diode operation with the corresponding equations was performed using the SPICE model $[8,9]$. Modeling of impedance and energy characteristics of passive elements involves determination of parameters of transmission line followed by its calculation with the node potential method. In that way we estimated, in particular, losses in the transmission line as well as developed topology of IC elements.

The substrate material was duroid with the following parameters: permittivity $\varepsilon_{r}=2.2$ and $\tan (\delta)=0.0009$. Using this material, we made a microstrip transmission line. To realize frequency multiplier, we developed the following elements: (i) filters for bringing diode bias voltage, (ii) a rejection filter for $280 \mathrm{GHz}$ and (iii) a microstrip line-to-rectangular waveguide transition for $280 \mathrm{GHz}$. The requirements to the filter for bringing diode bias voltage are such: (i) bringing voltage supply $\left(\mathrm{U}_{\text {bias }}\right)$ to the active element and (ii) microwave signal transmission from the input (RF In) to the output (RF Out) with minimal losses.

Fig. 2.1 shows topology of such filter. It is perceived that this filter will be set after the active element (IMPATT diode). The filter adds minimal losses at the frequency $280 \mathrm{GHz}$ or $360 \mathrm{GHz}$. The sizes of such filter elements for the frequencies chosen were obtained with the node potential method.

Since the metallization layer in such topology is continuous, bringing of a direct voltage or lowfrequency modulation signal from the input $U_{\text {bias }}$ occurs automatically. Another requirement is also fulfilled. An analysis shows that the losses in such filters are no more than $0.42 \mathrm{~dB}(0.32 \mathrm{~dB})$ at a frequency of $280 \mathrm{GHz}$ $(360 \mathrm{GHz})$.

The rejection filter topology is presented in Fig. 1.3a. This filter is intended for active element isolation from IC input at a high output frequency. 
Besides, it lets the master frequency through with minimal losses. The sizes of such filter elements for $280 \mathrm{GHz}$ were obtained with the node potential method. The losses in such filter are over $50 \mathrm{~dB}$ at the multiplier output frequency. At the same time, in both cases the added losses are no more than $0.5 \mathrm{~dB}$ at the reference oscillator frequency $(140 \mathrm{GHz})$.

Shown in Fig. 2.2 is a construction of a microstrip line-to-rectangular waveguide transition. Such transition ensures microwave signal transmission from the input (3) to the waveguide output (1) (cross-section of $0.8 \times 1.6 \mathrm{~mm}$ ) with minimal losses at the output frequency. The sizes of transition elements for $280 \mathrm{GHz}$ frequency were obtained with the node potential method [8]. Such transition adds losses no more than $0.5 \mathrm{~dB}$; this value is tolerable.

Fig. 2.3 shows topology of a frequency multiplier with SBD and all its elements. The purposes and parameters of separate parts were described above. This topology is used when designing photomasks intended for fabrication of IC test specimens. Shown in Fig. 2.4 is appearance of radiation source with frequency multiplier. Fig. 2.5 presents a block diagram of radiation source components with frequency of $280 \mathrm{GHz}$ at the multiplier. The technical characteristics of the radiation source with a frequency multiplier are given in Table.

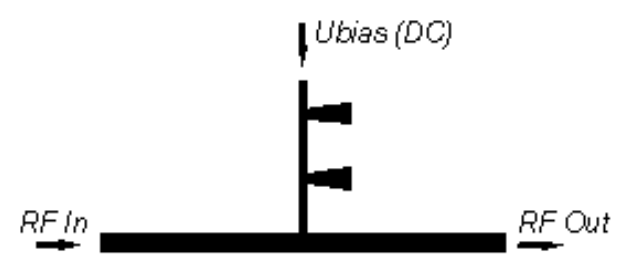

Fig. 2.1. Topology of a filter for supply of diode bias voltage [6].

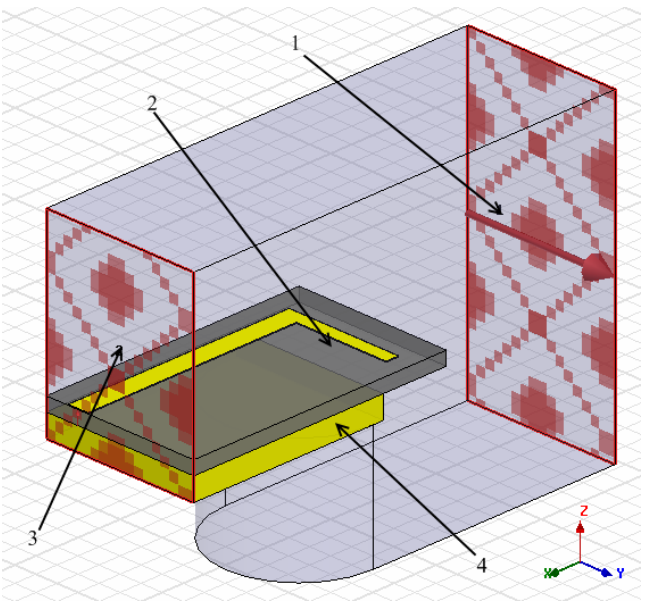

Fig. 2.2. Construction of a microstrip line-to-rectangular waveguide transition: 1 - rectangular waveguide section; $2-$ microstrip line-to-waveguide transition (excitation loop); 3 microstrip line intersection; 4 - microstrip line [6].

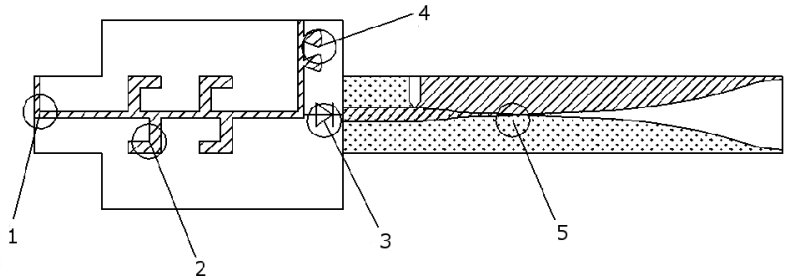

Fig. 2.3. Topology of a frequency multiplier: 1 - waveguide excitation loop at frequency multiplier output $(280 \mathrm{GHz}) ; 2$ rejection filter; 3 - SBD; 4 - supply filter; 5 - waveguide-tomicrostrip line transition for input signal $(140 \mathrm{GHz})[6]$.

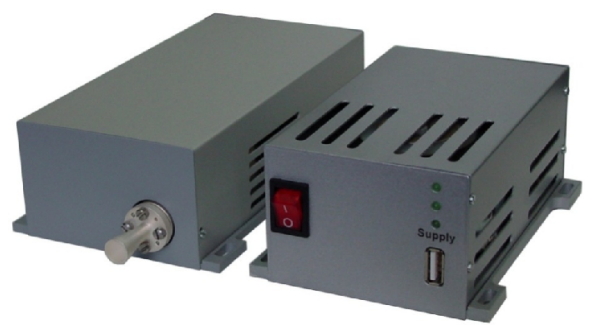

Fig. 2.4. Radiation source with frequency of $280 \mathrm{GHz}$ at the multiplier.

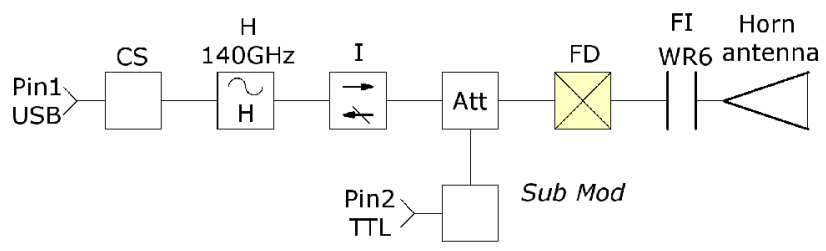

Fig. 2.5. Block diagram of radiation source components with multiplier frequency of $280 \mathrm{GHz}$ : CS - current source; H heterodyne with IMPATT diode, $P_{\text {out }}=30 \mathrm{~mW}$; I - isolator; Att - modulator with $p$ - $i-n$ diodes; FD - frequency doubler; Fl - outlet flange WR6, UG387; Sub Mod - submodulator.

Table. Source technical characteristics.

\begin{tabular}{|c|l|c|}
\hline$\#$ & \multicolumn{1}{|c|}{ Parameters } & Values \\
\hline 1 & Operating frequency, $\mathrm{GHz}$ & 280 \\
\hline 2 & Output power, $\mathrm{mW}$ & $0.3 \ldots 0.5$ \\
\hline 3 & $\begin{array}{l}\text { Amplitude modulation frequency, } \\
\mathrm{kHz}\end{array}$ & 1 \\
\hline 4 & Relative instability & $10^{-4} \ldots 10^{-5}$ \\
\hline 5 & Supply voltage, $\mathrm{V}$ & $220(50 \mathrm{GHz})$ \\
\hline 6 & Operating temperature range, ${ }^{\circ} \mathrm{C}$ & $+5 \ldots+40$ \\
\hline
\end{tabular}

\section{Subharmonic mixers with SBD for $220-325 \mathrm{GHz}$ and $325-400 \mathrm{GHz}$ operating frequency ranges}

The problem of development of receivers for submillimeter and $\mathrm{THz}$ regions is urgent. Such devices are per se heterodyne detectors whose most important element is frequency converter. The latter is a facility for transfer of signal spectrum to another frequency region,

\section{(C) 2014, V. Lashkaryov Institute of Semiconductor Physics, National Academy of Sciences of Ukraine}


with retention of the modulation law. To this end, a nonlinear element and local heterodyne are used to ensure operation mode of the conversion device. It is reasonable to use semiconductor SBDs as nonlinear elements in frequency converters. The slope of diode $I-V$ curves varies with a rather high signal $U_{H}$ from heterodyne and remains practically the same under action of a low signal $U_{s} \quad\left(U_{s}<<U_{H}\right)$. Frequency multiplication is realized owing to the signal effect of on the slope of $I-V$ curve of diode that is a part of mixer.

Stability of both frequency and power level of heterodyne is a significant requirement. The problem of development of highly stable heterodyne for frequencies over $200 \mathrm{GHz}$ and required power level is rather complicated. But it is possible to use the mixer property to operate at a heterodyne frequency half as high as useful signal. A mixer with subharmonic pumping that operates at the second harmonic of heterodyne signal makes it possible to halve the signal operating frequency and thus to essentially simplify realization of the required power level at a lower frequency.

By applying the four-terminal network theory and analyzing operation of transceiver radio channel, the following conclusions have been drawn: (i) to minimize receiver noise factor, one should ensure maximization of transmission coefficient and minimization of mixer noise factor; (ii) to increase power level of transmitter signal, one should maximize transmission coefficient. Thus, taking into account that conversion losses of a passive mixer are approximately equal to the noise factor, the main criterion for design of mixer is minimization of conversion losses.

We used the hybrid mixer topology and chose for nonlinear element an SBD with beam leads obtained by metal layer deposition onto semiconductor surface $[10,11]$. This diode has quadratic $I-V$ curve and the lowest loss resistance among the diodes used when making microwave mixers [12, 13]. For the chosen diodes of different capacitances we measured $C-V$ curves (Fig. 3.1a) and $I-V$ curves at the forward branch (Fig. 3.1b). Fig. 3.1a shows that in SBD for mixers (contrary to the frequency multiplier diodes) $C-V$ curves are practically straight line. The loss currents did not exceed $1 \mathrm{nA}$.

Mathematical modeling of SBD was performed using the SPICE model. The latter attributes diode $I-V$ curve to its physical parameters. A diode SPICE model involves a number of mathematical functions that describe diode operation at different initial parameters. SBD (at which a wanted signal and that from the heterodyne come) is approximated by an equivalent circuit, with account made for the initial parameters. An analysis is made by constructing equation for circuit using the modified node potential method [9]. The initial diode parameters were chosen from the experimental $C-V$ curves and $I-V$ curves.

We performed modeling of board topology for microwave mixers with a rejection filter and waveguide- to-microstrip line tapered transition using the node potential method. The general appearance of board for microwave mixers is presented in Fig. 3.2.

Based on mathematical modeling of diode operation and 3D numerical modeling of electromagnetic field in the mixer made with the node potential method, we developed mixer test specimens for the $220-325 \mathrm{GHz}$ and $325-400 \mathrm{GHz}$ frequency ranges. Shown in Figs 3.3a and 3.3b are appearances of mixer test specimens.

The mixers involve a case, board and microwave mixer SBD with beam leads. A board and microwave SBD are mounted in the mixer waveguide channel. The board material is quartz $25 \mu \mathrm{m}$ thick, metallized on both sides. The metallization thickness was $3 \mu \mathrm{m}(1 \mu \mathrm{m})$ for the $220-325 \mathrm{GHz}(325-400 \mathrm{GHz})$ frequency range. For the $220-325 \mathrm{GHz}$ frequency range, the cross-section of waveguide channel of heterodyne input (signal input) is $1.2 \times 2.4 \mathrm{~mm}(0.4 \times 0.8 \mathrm{~mm})$, while for the $325-400 \mathrm{GHz}$ frequency range, the corresponding cross-section is $0.8 \times 1.6 \mathrm{~mm}(0.36 \times 0.71 \mathrm{~mm})$.
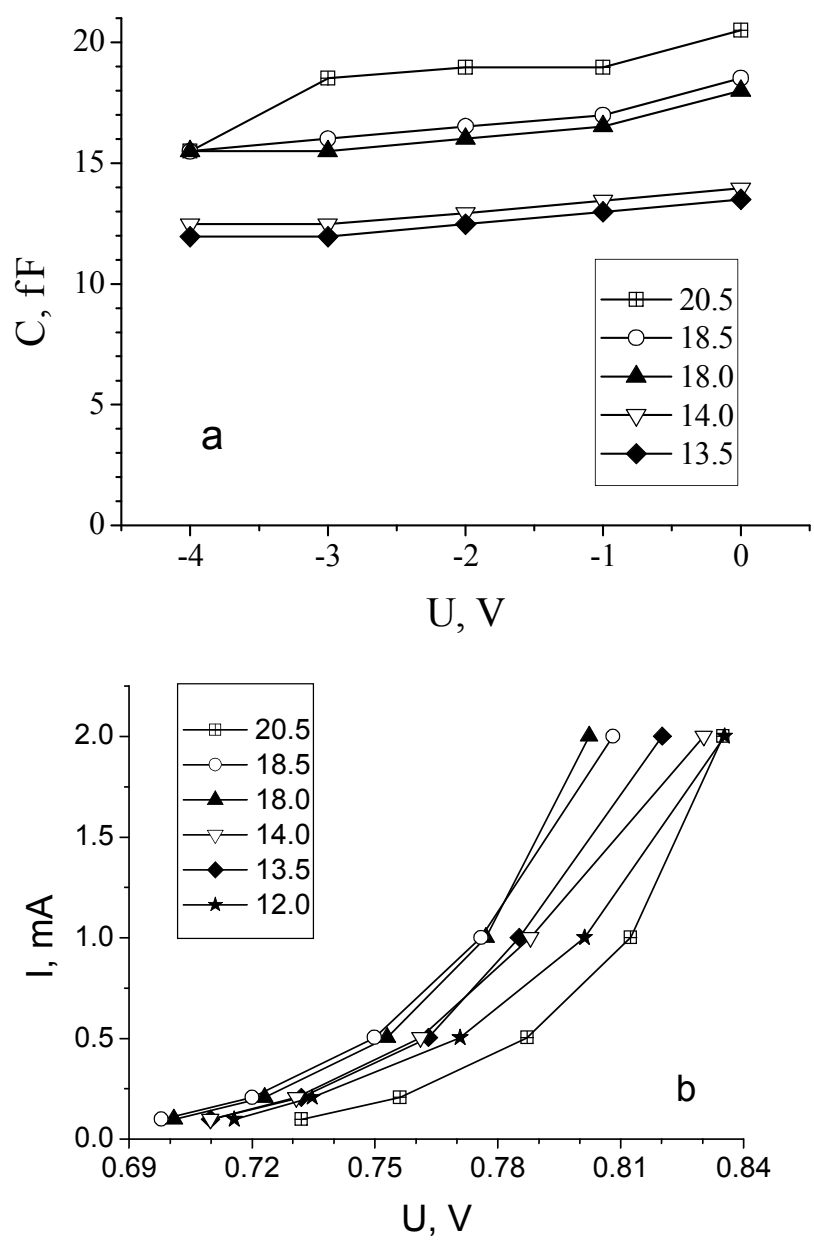

Fig. 3.1. a) $C-V$ curves of SBD with different values of metal-semiconductor contact capacitance $C_{\mathrm{jo}}$ (in femtofarads see inset); b) $I-V$ curves of SBD with different values of metal-semiconductor contact capacitance $C_{\mathrm{jo}}$ (in femtofarads see inset). 
We studied the electric parameters of the developed mixers. The results of measurement of conversion losses as a function of heterodyne power are presented in Figs $3.4 \mathrm{a}$ and $3.4 \mathrm{~b}$. One can see that conversion losses $K$ firstly decrease as heterodyne power $\left(P_{H}\right)$ grows and then this trend becomes slower. For subharmonic mixers for the $220-325 \mathrm{GHz}$ frequency range the conversion losses are below $30 \mathrm{~dB}$ at the maximal heterodyne power of $25 \mathrm{~mW}$, while for subharmonic mixers for the 325$400 \mathrm{GHz}$ frequency range the conversion losses begin to flatten out at heterodyne power over $15 \mathrm{~mW}$ and are below $30 \mathrm{~dB}$ at the maximal heterodyne power of $18 \mathrm{~mW}$. Further grows of heterodyne power leads to burning out of SBD with beam leads [9].

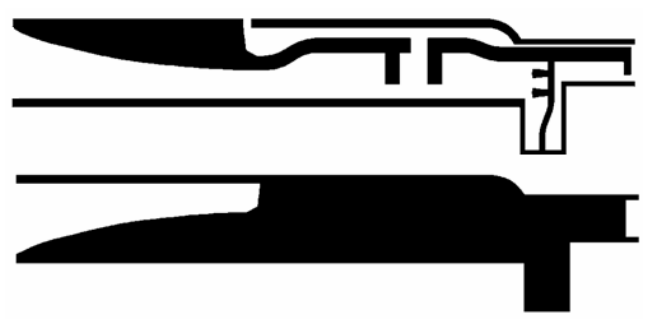

Fig. 3.2. Microwave mixer board.
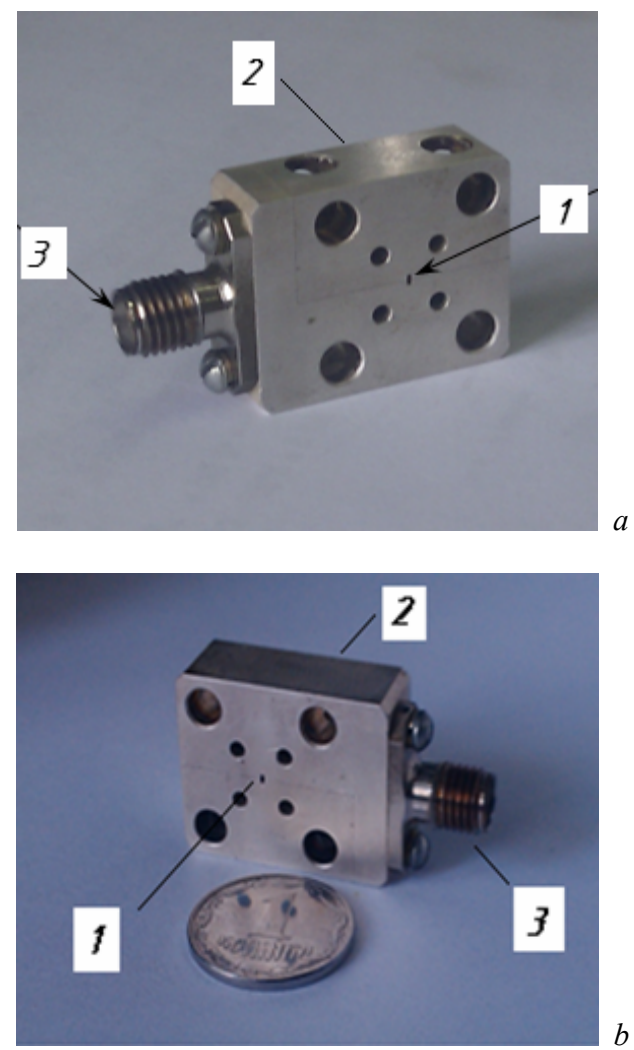

Fig. 3.3. Mixer test specimens for $220-325 \mathrm{GHz}$ frequency range [14]. (a): 1 - waveguide input of signal $(0.4 \times 0.8 \mathrm{~mm}) ; 2$ - waveguide input of heterodyne $(1.2 \times 2.4 \mathrm{~mm}) ; 3$ intermediate frequency (IF) and $325-400 \mathrm{GHz}$ frequency range. (b): 1 - waveguide input of signal $(0.36 \times 0.71 \mathrm{~mm}) ; 2$ waveguide input of heterodyne $\left(0.8 \times 1.6 \mathrm{~mm}^{2}\right) ; 3$ - IF output.
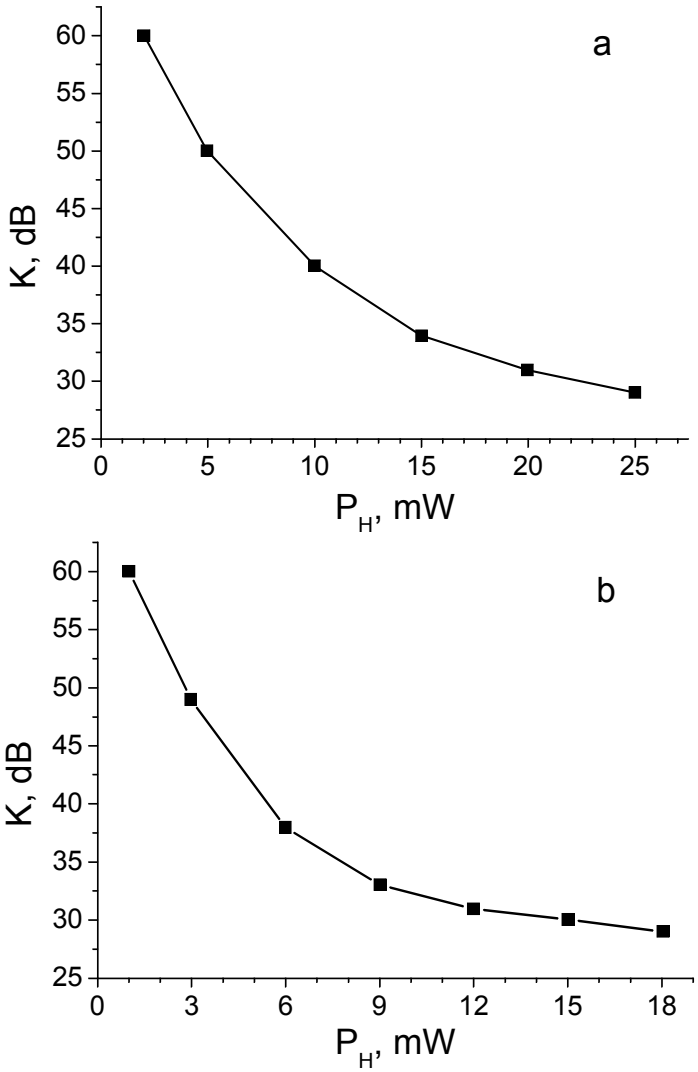

Fig. 3.4. Subharmonic mixer conversion losses $K$ as a function of heterodyne power $P_{H}$ : a) for $220-325 \mathrm{GHz}$ range at a frequency of $280 \mathrm{GHz}$ [14] and b) for $325 \div 400 \mathrm{GHz}$ range at a frequency of $360 \mathrm{GHz}$.

\section{Conclusions}

The following equipment has been made in the course of execution of the research project according to the Government Special-Purpose Scientific and Technical Program "Nanotechnologies and Nanomaterials" in 2010-2013:

- detector sections for the $220-325 \mathrm{GHz}$ and 325 $400 \mathrm{GHz}$ frequency ranges that ensure conversion factor within $1000-115 \mathrm{~V} / \mathrm{W}$ and $120-102 \mathrm{~V} / \mathrm{W}$, respectively;

- radiation source using master-oscillator with a silicon IMPATT diode (operating frequency of $140 \mathrm{GHz}$ ) and frequency doubler with SBD (operating frequency of $280 \mathrm{GHz}$ and output power of $0.3-0.5 \mathrm{~mW}$ );

- subharmonic mixers for the $220-325 \mathrm{GHz}$ and $325-400 \mathrm{GHz}$ frequency ranges, with conversion losses no more than $30 \mathrm{~dB}$.

\section{References}

1. K.R. Jha, G. Singh, Terahertz planar antennas for future wireless communication: A technical review // Infrared Physics \& Technology, 60, p. 71-80 (2013). 
2. M. Koch, Terahertz technology: A land to be discovered // Optics and Photonics News, 18(3), p. 20-25 (2007).

3. A.G.U. Perera, G. Ariyawansa, S.G. Matsik, Terahertz detection devices, in: Comprehensive Semiconductor Science and Technology. Elsevier, Amsterdam, 6, p. 265-307 (2011).

4. A. Rogalski, F. Sizov, Terahertz detectors and focal plane arrays // Optoelectronics Rev. 19(3), p. 79137 (2011).

5. M. Ghanashyam Krishna, Sachin D. Kshirsagar, Surya P. Tewari, Terahertz emitters, detectors and sensors: Current status and future prospects, in: Photodetectors. Ed. by Sanka Gateva, InTech, 2012, p. 115-144.

6. A.V. Zorenko, Ya.Ya. Kudryk, Yu.V. Marunenko, Development and investigation of microwave radiation sources and detector sections using SBDs within the 220-400 $\mathrm{GHz}$ frequency range // Semiconductor Physics, Quantum Electronics \& Optoelectronics, 14(4), p. 411-415 (2011).

7. R.R. Vakhitov, Digital Frequency Multipliers, Candidate of Technical Sci. Dissertation 05.13.05 (fundamental theory, mathematical models, investigation, development). Ufa, 2006 (in Russian).

8. J. Grajal, V. Krozer, E. González, F. Maldonado, J. Gismero, Modeling and design aspects of millimeter-wave and submillimeter-wave Schottky diode varactor frequency multipliers // IEEE Trans. on Microwave Theory and Techniques, 48(4), p. 700-711 (2000).
9. A. Gutin, T. Ytterdal, V. Kachorovskii, A. Muraviev, M. Shur, THz SPICE for modeling detectors and nonquadratic response at large input signal // IEEE Sensors J. 13(1), p. 55-62 (2013).

10. E.P. Groo, M.G. Ignatiev, L.A. Kozlova, T.C. Petrova, Technological aspects of a GaAsbased microwave mixer SBD, in: Modern Problems in Radio Electronics: A Collection of Scientific Papers. Ed. by A.I. Gromyko, A.V. Sarafanov, IPTs KGTU, 2005, p. 327 (in Russian).

11. B. Thomas, A. Maestrini, G. Beaudin, A lownoise fixed-tuned $300-360-\mathrm{GHz}$ sub-harmonic mixer using planar Schottky diodes // IEEE Microw. Wirel. Compon. Lett. 15(12), p. 865-867 (2005).

12. M.E. Belkin, L.M. Belkin, Features of constructing monolithic version of mm-wave resistive mixers // Elektronnaya Tekhnika, Ser. 2 "Poluprovodnikovye Pribory”, no 1, p. 98-104 (2010), in Russian.

13. N.R. Erickson, T.M. Goyette, TeraHertz Schottkydiode balanced mixers // Proc. SPIE, 7215, Terahertz Technology and Applications II. Ed. by K.J. Linden, L.P. Sadwick, C.M.M. O'Sullivan, 721508 (2009).

14. A.V. Zorenko, N.V. Kolesnik, T.V. Kritskaya, Ya.Ya. Kudryk, Yu.V. Marunenko, L.P. Ryzhanovich, A subharmonic mixer for the $220-325 \mathrm{GHz}$ frequency range // Semiconductor Physics, Quantum Electronics \& Optoelectronics, 16(1), p. 84-85 (2013). 